\title{
Aktive Lohnpolitik in Europa
}

\author{
JÖRG HOFMANN
}

Als Reaktion auf die Wirtschafts- und Finanzmarktkrise, die 2008 auch Europa erfasste, etablierte die Europäische Kommission gemeinsam mit den Regierungen der EU-Mitgliedstaaten ein neues System der wirtschaftlichen Steuerung (Economic Governance). So richtig der Ausbau einer gemeinsamen Wirtschaftspolitik und hierfür geeigneter Instrumente und Methoden ist, führte dies im Kontext einer einseitigen Austeritätspolitik doch zu einer deutlichen Krisenverschärfung. Das neoliberale Dogma einer rein auf preisliche Wettbewerbsfähigkeit angelegten Wirtschaftspolitik macht Löhne und Sozialleistungen zur zentralen Anpassungsvariable der derzeit noch bestehenden makroökonomischen Ungleichgewichte. Die Gewerkschaften in vielen Ländern Europas sehen sich einer interventionistischen „brutalen“ Lohnkoordinierung nach unten ausgesetzt, die unter Missachtung der Tarifautonomie auf die Destabilisierung von Tarifbindung und Eingriffe in Tarifniveaus zielt. Damit wird nicht nur eine makroökonomische Geisterfahrt durchgesetzt, sondern auch ein wesentliches Freiheitsrecht eingeschränkt. Die Resultate dieser Politik werden immer offensichtlicher:

- Steigende Arbeitslosigkeit vor allem in den Ländern Süd- und Südosteuropas mit Spitzenwerten von $25,8 \%$ in Spanien und 27,8\% in Griechenland im Jahr 2013;

- sinkende Reallöhne in zwei Dritteln der EUMitgliedstaaten durch Eingriffe in Tarifvertragssysteme und Entgeltkürzungen;

- gestiegenes Armutsrisiko vor allem auch von Beschäftigten in Vollzeit und mit höherem Ausbildungsniveau.

All dies führt in den betroffenen Ländern zu einem massiven Einbruch in der gesamtwirtschaftlichen Nachfrage. Selbst einzelne Generaldirektionen der Kommission erkennen neuerdings die Risiken, die mit einer unveränderten „Weiter-sowie-bisher-Politik“ verbunden sind. Die Generaldirektion Beschäftigung, Soziales und Integration sieht die soziale Kohäsion der Europäischen
Union in Gefahr. Und auch die Generaldirektion Wirtschaft und Finanzen - bisher entschiedenste Verfechterin der angebotsorientierten Ausrichtung der Reformpolitik - gibt mittlerweile kritischere Einschätzungen zu den Nebeneffekten der Konsolidierungsprogramme, die die Rezession in den betroffenen Ländern weiter verschärfen. ${ }^{1}$

In Abgrenzung zum bisherigen Kurs in der EU könnte eine nachfrageorientierte Wachstumspolitik mit stärkeren Entgeltsteigerungen und öffentlichen Investitionen in Europa entscheidend zur Stabilisierung beitragen. Dem stehen allerdings auch die politischen Mehrheitsverhältnisse in vielen europäischen Ländern - gerade in den Krisenstaaten - entgegen. Einen zweiten Ansatzpunkt bietet die Lohnpolitik: Der angebotsorientierten Lohnkoordinierung muss eine nachfrageorientierte Lohnkoordinierung entgegengesetzt werden. Deren Träger können allein die Gewerkschaften sein, flankiert durch staatliches Handeln: gegen prekäre Beschäftigung und für die (gesetzliche) Stärkung von Tarifautonomie und Tarifbindung. Ein langfristiger Erfolg dieses Politikansatzes wird von der europäischen Koordinierung bestimmt. Eine europäische Koordinierung der Tarifpolitik zwischen den nationalen Gewerkschaftsorganisationen ist daher nach wie vor von hoher Aktualität.

\section{Grundgedanke der europäischen Koordinierung der Tarifpolitik}

Die Ursprünge und Beschlüsse zu einer europäischen Lohnkoordinierung des Europäischen Metallgewerkschaftsbundes (EMB), seit Mai 2012 in die europäische Industriegewerkschaftsföderation IndustriAll-Europe aufgegangen, liegen weit vor Ausbruch der aktuellen Wirtschaftskrise in Europa. Angesichts der Einführung der gemeinsamen europäischen Währung im Jahr 1999 bekräftigten die Mitgliedsgewerkschaften des EMB ein Jahr zuvor auf der 3. Tarifpolitischen Konferenz in Frankfurt am Main die Notwendigkeit einer europäischen Koordinierung der Tarifpolitik, um Unterbietungs- und Absenkungskonkurrenz zu verhindern. Der EMB und seine
Mitgliedsgewerkschaften beschlossen eine Lohnkoordinierungsregel, die bis heute gilt. Fast 16 Jahre später ist angesichts der aktuellen Austeritätspolitik die damalige Feststellung des EMB im Zusammenhang mit der Koordinierung der Tarifpolitik noch immer aktuell: „Die Verbesserung der Wettbewerbsfähigkeit darf nicht über soziales Dumping mit einer Verschlechterung der Arbeits- und Lebensbedingungen erkauft werden. Die Verbesserung der Wettbewerbsfähigkeit muss vielmehr mit der Verbesserung der Arbeitsund Lebensbedingungen einhergehen. ${ }^{\text {“2 }}$

\section{Aktuelle Herausforderungen für die europäische Tarifpolitik}

Die unheimliche Allianz, die sich zwischen den Guidelines der Troika und dem politischen Wollen konservativ-neoliberaler Regierungen in den Krisenstaaten herausgebildet hat, rüttelt an den Grundfesten gewerkschaftlicher Gestaltungsmacht: der Tarifautonomie und der Tarifbindung. Die EU-Kommission gibt offen zu, Maßnahmen zu fördern, „die zu einer generellen Reduzierung der Lohnsetzungsmacht von Gewerkschaften führen. ${ }^{\text {“3 } B e s o n d e r s ~ b e t r o f f e n ~ s i n d ~ L a ̈ n d e r ~ w i e ~}$ Griechenland, Portugal und Spanien, die bisher eine vergleichsweise hohe Tarifbindung von 80 bis $90 \%$ hatten. Diese wurde jedoch überwiegend durch Allgemeinverbindlicherklärungen bzw. Erga-Omnes-Regelungen ${ }^{4}$ erreicht. Die Ver-

1 Vgl. European Commission, Directorate-General for Economic and Financial Affairs (2013): Fiscal consolidations and spillovers in the Euro area periphery and core, Economic Paper (506), Brussels.

2 Europäischer Metallgewerkschaftsbund (EMB) (2006): Meilensteine, Brüssel, S. 263.

3 European Commission (2012): Labour Market Developments in Europe 2012, Brussels, S. 104.

4 In einer Vielzahl europäischer Länder existieren (anders als in Deutschland, wo die Tarifautonomie durch das Grundgesetz geschützt ist) gesetz- 
wundbarkeit einer solcher Art erzielten hohen Tarifbindung liegt klar auf der Hand: Sie kann jederzeit per Gesetzesänderung ausgehebelt werden. Aktuell wird unter den Mitgliedsgewerkschaften von IndustriAll-Europe eine Debatte zur Entwicklung einer Strategie zur Erhöhung der Tarifbindung geführt. Die IG Metall setzt sich nachdrücklich für den Weg der Stärkung der Durchsetzungsmacht durch Mitgliederzuwachs ein.

Aber ohne Zweifel ist auch in Deutschland ein gesetzlicher Rahmen zur Verbreiterung von Tarifbindung und tariflichen Mindeststandards in einem wachsenden Sektor faktischer Tariflosigkeit wichtig. Daher weist die aktuelle Gesetzgebung zur Stärkung der Tarifautonomie in die richtige Richtung. Dies gilt umso mehr für Länder, in denen Verhandlungstraditionen sich stark an staatlicher Regulierung des Tarifvertragssystems und nicht an Mitgliederstärke orientierten.

Eine weitere Ursache der negativen Lohndrift ist der massive Ausbau prekärer Beschäftigungsverhältnisse in Europa. Partiell kann dies durch tarifpolitische Regelungen aufgefangen werden, wie dies der IG Metall 2012 gelungen ist. Dennoch ist hier auch die Arbeitsmarktpolitik gefordert. Die Mitgliedsgewerkschaften von IndustriAll-Europe bündeln diesbezüglich ihre Aktivitäten unter der Gemeinsamen Forderung: „Für sichere Arbeitsplätze, gegen prekäre Arbeit“.

Als Folge der Deregulierung der Arbeitsmärkte und von Interventionen in Tarifvertragssysteme lässt sich ein grundlegend gewandeltes Muster in der europäischen Lohnentwicklung ablesen. Im Zeitraum 2001 bis 2009 verzeichneten fast alle EU-Mitgliedstaaten ein positives Reallohnwachstum. 2010 bis $2013 \mathrm{kam}$ es in nur noch wenigen Ländern zu - zumeist moderaten - Reallohnsteigerungen. Und in 18 von 27 EU-Staaten sind die Reallöhne zurückgegangen. So ist eine europaweite Lohnsenkungsspirale in Gang gekommen, die den privaten Konsum reduziert, deflationäre Tendenzen fördert und die ökonomische Stagnation Europas zementiert.

Angemerkt muss werden, dass sich diese Entwicklung in Deutschland nicht für alle Beschäftigten gleich darstellt. Konnten die Beschäftigten in tarifgebundenen Betrieben des wettbewerbsintensivsten Sektors, der Exportindustrie, einen deutlich über der Inflation und dem Produktivitätswachstum liegenden Lohnzuwachs und damit ein Mehr an Kaufkraft erzielen, ging die intersektorale Lohnschere weiter auf. Insbesondere in den typischen Niedriglohnbereichen personenbezogener Dienstleistungen wurde dieses Lohngefäl- le auch durch die Prekarisierung von Arbeitsverhältnissen befördert. Und insgesamt spielt die nachlassende Tarifbindung in allen Sektoren eine große Rolle. Zur Analyse und der Entwicklung notwendiger Schlussfolgerungen sind daher Mittelwerte der nationalen Lohnentwicklung alleine nicht geeignet. Es bedarf ergänzender inter- und intrasektoraler Analysen.

\section{Lohnkoordinierung in Europa weiterentwickeln}

Die durch die Interventionen in die Tarifverhandlungssysteme vorangetriebene Dezentralisierung der Tarifpolitik droht die Gewerkschaften in der Governance europäischer Wirtschaftspolitik zu marginalisieren, steuern sie dem nicht entgegen. Um als wesentlicher Player in einer europäischen Geld-, Finanz- und Wirtschaftspolitik mitzuwirken, ist eine weitergehende Kooperation und Koordination erforderlich. Mit der Gründung von IndustriAll konnte eine wesentliche Voraussetzung für eine intensivere Kooperation der Gewerkschaften des Industriesektors in Europa geschaffen werden; deren Vorzüge müssen jetzt genutzt werden.

Eine europäische Koordinierung der Tarifund insbesondere der Lohnpolitik ist nach wie vor von hoher Aktualität. Dieses Feld können Gewerkschaften nicht der EU-Kommission überlassen. Die Gewerkschaften müssen dem europäischen Semester der Kommission - mit sich jährlich wiederholenden Berichten zu ökonomischen Kennziffern - ein ebenso verbindliches europäisches Semester der Gewerkschaften, also ein System sozialer Kennziffern und konkreter Zielsetzungen der Lohnpolitik, gegenüberstellen.

Dies bedeutet allerdings nicht, sich auf den von der Kommission gesetzten engen institutionellen Rahmen einzulassen. Denn mit der Lohnpolitik verfügen Gewerkschaften über ein autonomes Instrument, um auf Verteilung, Nachfrage und Beschäftigung Einfluss zu nehmen. Sie müssen daher die eigenen Kriterien der europäischen Koordinierung der Tarifpolitik weiterentwickeln. Erste Schritte einer solchen Weiterentwicklung finden sich in der 2012 von IndustriAll-Europe übernommenen und weiterentwickelten Lohnkoordinierungsregel. Die Diskussion hierzu muss weitergeführt werden.

Die jetzige Koordinierungsregel ist ausreichend für gereifte Volkswirtschaften mit geringen sektoralen Produktivitätsdifferenzen und einer stabilen Preis- und Beschäftigungssituation. Diese Voraussetzungen werden wir längere Zeit nicht vorfinden. Insoweit müsste die Koordinierungsregel unterschiedliche Situationen adäquat abbilden, z. B. für Länder im Krisenmodus oder Länder mit schnellem strukturellen Wandel und hohen positiven Produktivitätsdifferenzen des industriellen Sektors.

So hat die IG Metall seit Beginn des Jahrtausends ihre lohnpolitischen Koordinaten fortentwickelt und begründet ihre Lohnpolitik mit der Zielinflationsrate der Europäischen Zentralbank (EZB), der mittelfristigen Produktivitätsentwicklung und einem Umverteilungsfaktor, der als Korrekturfaktor verteilungspolitische Fehlentwicklungen korrigieren soll. Evident wird die Verwendung der Zielinflationsrate bei drohenden Deflationstendenzen, wie wir sie aktuell beobachten. Offensichtlich ist aber auch, dass Reallohnsicherung als Ziel gewerkschaftlicher Lohnpolitik gesetzt ist.

$\mathrm{Zu}$ Zielkonflikten führt dies dort, wo die Inflationsrate deutlich über der Zielinflationsrate und nationaler Produktivitätsentwicklung liegt, wie es früher für die Krisenländer eher typisch war. Aktuell haben wir aber auch in diesen Ländern deflationäre Tendenzen. Offenkundig ist auch, dass die alleinige Bezugnahme auf die nationale Produktivitätsentwicklung in den „Aufholländern" Osteuropas nicht sinnvoll ist. Die dort erzielten Produktivitätssprünge in der Industrie führen zu einer verteilungspolitischen Schieflage, wenn sie sich nicht auch in der Lohnentwicklung der in diesem Sektor Beschäftigten wiederfinden.

Insbesondere muss auch die praktische Umsetzung einer Koordinierung in den europäischen Gewerkschaften deutlich verbessert werden. Es stellt sich die Frage einer Verbindlichkeit von Koordination. Das wiederum setzt voraus, dass sich mehr europäische Gewerkschaften aktiv beteiligen. Hier sind die Schwächen offensichtlich. Das liegt zum Teil an fehlenden Ressourcen, aber vor allem an der geringen Priorität, die eine europäische Koordinierung in einzelnen Gewerkschaften hat. Eine Revitalisierung der Debatte um die Notwendigkeit europäischer Koordinierung der Tarifpolitik, wie sie die Phase

liche Erga-Omnes-Regelungen. „Dies bedeutet dass die Tarifbestimmungen in tarifgebundenen Unternehmen automatisch auch für nicht-organisierte Beschäftigte gelten." (Schulten, Th. (2012) Stellenwert der Allgemeinverbindlicherklärung für die Tarifvertragssysteme in Europa, in: WSIMitteilungen 65 (7), S. 486, http://www.boeckler. de/wsimit_2012_07_schulten.pdf). 
in Vorbereitung der Euro-Einführung kennzeichnete, ist dringend notwendig. Die aktuelle Situation bietet dafür Anlass genug.

Wollen die Gewerkschaften mit ihrer Tarifpolitik im Rahmen der europäischen Governance eine aktive Rolle spielen, müssen sie ihre Ziele und Koordinaten transparent machen und ihre Fähigkeit unter Beweis stellen, gemeinsame Koordinierungsregeln auch zu befolgen.

IndustriAll-Europe sollte weiterhin als Orientierungshilfe für Tarifverhandlungen der Mitgliedsorganisationen Prognosen zur Produktivitäts- und Inflationsentwicklung für das jeweils kommende Jahr für jedes Herkunftsland der Mitgliedsorganisationen zusammenstellen und veröffentlichen. Auf dieser Basis können tarifpolitische Ziele durch die Einzelgewerkschaften begründet und transparent gemacht werden.

Ob diese Ziele erreicht werden, kann kein statistischer Vorgang sein, der Abweichungen von einer Regel positiv oder negativ bewertet. Vielmehr gilt es, die Gründe der Abweichungen $\mathrm{zu}$ analysieren und transparent $\mathrm{zu}$ machen, um gemeinsame Strategien zu entwickeln, die dem entgegenwirken. Und es geht auch darum, die europäische Politik sichtbar und nachvollziehbar zu machen. Zudem müssen die Entwicklung der langfristigen Verteilungsrelationen, die unterschiedlichen wirtschaftlichen und strukturellen Voraussetzungen der jeweiligen Länder wie auch die Entwicklung von Organisationsmacht und Arbeitsmarkt berücksichtigt werden.

Diese Grundstruktur einer europäischen gewerkschaftlichen Governance scheint mir wesentlich, aber auch sehr voraussetzungsvoll, um der Marginalisierung der Gewerkschaften und ihres Einflusses in Europa und seinen Institutionen entgegenzuwirken. Es gilt, das System der solidarischen Tarifpolitik als Kern einer auf Subsidiarität bauenden Gestaltung der Arbeits- und Wirtschaftsbedingungen in Europa zu erhalten. Organisationsmacht und Tarifbindung sind unverzichtbare Voraussetzungen für die Ausschöpfung des Verteilungsspielraumes und damit auch für die Erfüllung der Lohnkoordinierungsregel.

\section{Grenzen der europäischen Tarifpolitik zur Krisenbewältigung}

Die Tarifpolitik kann die aktuelle europäische Krise jedoch sicher nicht alleine lösen. Die derzeit dominante Perspektive reduziert den Hauptgrund für die makroökonomischen Ungleichgewichte auf die Lohnentwicklung in den Ländern der Euro-Zone. Außer Acht gelassen werden Ursachen, die in der Wirtschaftsstruktur der
Länder und im unterschiedlichen Ausgangsniveau bei Beitritt zur Währungsunion liegen.

Die Industriestruktur der Länder ist in der Debatte um die Leistungsbilanzdefizite und -überschüsse eine entscheidende Größe. Hoch entwickelte Volkswirtschaften wie die Bundesrepublik Deutschland exportieren zum Großteil hoch spezialisierte Industriegüter, die schwer substituierbar sind und deren Nachfrage relativ unabhängig von der Preisentwicklung ist. Nicht-preisliche Wettbewerbsfaktoren wie Produktqualität und -komplexität, Innovationsfähigkeit, Liefertreue, Servicepakete sowie der Konjunkturverlauf in den jeweiligen Exportmärkten sind bei diesen preisunelastischen Industriegütern entscheidend. Neuere Untersuchungen der Europäischen Kommission machen für den gesunkenen Marktanteil der Industriegüter Frankreichs und Italiens seit dem Jahr 2000 ein Zurückfallen bei den nicht-preislichen Wettbewerbsfaktoren gegenüber den deutschen Industriegütern verantwortlich. ${ }^{5}$

Wird dies berücksichtigt, so wird im Umkehrschluss schnell klar, dass eine Absenkung der Löhne und Gehälter in den Defizitländern nicht zu einer stabilen wettbewerbsfähigen wirtschaftlichen Basis führt. Die muss über eine europäische Industriepolitik geschaffen werden, wie sie beispielsweise im Programm „Ein neuer Weg für Europa“ vom Europäischen Gewerkschaftsbund gefordert wird. Dieses Programm enthält u.a. die Forderung nach zusätzlichen jährlichen Investitionen in Höhe von $2 \%$ des in der EU erzielten Bruttoinlandsprodukts (BIP) über einen Zeitraum von zehn Jahren, die in Zukunftsbereiche wie nachhaltige Energieproduktion und Energieeffizienz, moderne Verkehrsinfrastruktur, Erneuerung der industriellen Basis, Bildung und Ausbildung geleitet werden sollen. Hierzu gehört aber auch die Einbeziehung der Beschäftigten und ihrer Interessensvertretungen in die Gestaltung dieses strukturellen Wandels. Eine europäische Mitbestimmungsinitiative ist gefordert.

An einer Weiterentwicklung des geeinten und demokratischen Europa zu einer Währungs-, Wirtschafts- und Sozialunion mit demokratisch legitimierten Entscheidungsstrukturen führt kein Weg vorbei. Vielmehr müssten die Konstruktionsfehler der heutigen europäischen Governance beseitigt werden. Einer dieser

5 Vgl. European Commission, DG Economic and Financial Affairs (2012): Macroeconomic imbalances - France, Occasional Papers (105), July, Brussels, S. $22 \mathrm{f}$.
Konstruktionsfehler ist die nur randständige Berücksichtigung der zivilgesellschaftlichen Akteure wie Tarifvertragsparteien in der europäischen Governance. Der „soziale Dialog“ kann diese Lücke nicht schließen.

Wir sollten daher als Gewerkschaften auf eine autonome europäische Governance setzen, wollen wir die Tarifautonomie als Freiheitsgut vor interventionistischen Eingriffen schützen. Gleiches gilt auch für die Arbeitgeberverbände. Die nationalen Politiken wie auch die europäische Politik müssen diesen Prozess stützen. Die von den Gewerkschaften geforderte und nun von der Politik aufgenommene Stärkung von Tarifautonomie und Tarifbindung in Deutschland ist von daher ein ermutigendes Zeichen auch für Europa.

\section{AUTOR}

JÖRG HOFMANN ist Zweiter Vorsitzender der IG Metall und als Vorstandsmitglied zuständig für Tarifpolitik, Betriebs- und Branchenpolitik.

joerg.hofmann@igmetall.de 